\title{
CHIP ANTENNA FOR 5.2 GHZ WIRELESS LAN
}

\author{
Mitsuo TAGUCHI* Kazuhiro ICHIKAWA** Hideaki SHIMODA*** Kazumasa TANAKA* \\ * Dept. of Electrical \& Electronic Eng., Nagasaki University \\ ** Graduate School of Science and Technology, Nagasaki University \\ 1-14 Bunkyo-machi, Nagasaki-shi, 852-8521 JAPAN \\ *** Technical Center, TDK Co. \\ 2-15-7 Higashi-Ohwada, Ichikawa-shi, Chiba, 272-8558 JAPAN \\ *mtaguchi@net.nagasaki-u.ac.jp
}

\section{INTRODUCTION}

The authors have proposed two types of microstrip antennas printed on the parallelepiped dielectric chip for the Bluetooth system and the $5.2 \mathrm{GHz}$ wireless LAN. These antennas are excited by the electromagnetic coupling. For the Bluetooth system, the rectangular patch conductor is printed on the chip and excited by the monopole antenna printed on the sidewall of chip [1]. The size of dielectric chip is $12 \mathrm{~mm}$ by $12 \mathrm{~mm}$ by $4 \mathrm{~mm}$. For the $5.2 \mathrm{GHz}$ Wireless LAN, the shorted rectangular patch microstirp antenna is excited by the three monopoles composed of through holes within the chip [2]. The size of this antenna is $8 \mathrm{~mm}$ by $6 \mathrm{~mm}$ by $2 \mathrm{~mm}$.

In this paper, the chip antenna for $5.2 \mathrm{GHz}$ wireless LAN is numerically and experimentally analyzed and the reason for its small size is discussed. In the numerical analysis, the electromagnetic simulator "Fidelity" based on the FDTD method is used [3].

\section{ANALYTICAL MODEL}

Figure 1(a) and (b) show the structure of chip antenna. The patch conductor is printed on the surface of chip and the patch is short-circuited at the opposite side of the feed monopoles. This antenna is excited by three monopole antennas connected to the microstrip line on the lower substrate. The relative permittivity of lower dielectric substrate is $\varepsilon_{r 1}=4.4$ and that of upper chip is $\varepsilon_{r 2}=3.9$. For the space diversity, two chip antennas are mounted on the lower dielectric substrate.

In the numerical analysis by Fidelity, the calculation region is $75 \mathrm{~mm}$ by $75 \mathrm{~mm}$ by $45 \mathrm{~mm}$ in dimensions. The space steps are from $0.1 \mathrm{~mm}$ to $1 \mathrm{~mm}$ (non-uniform mesh). The perfectly matched layer of six-layer and fourth-order is used as the absorbing boundary condition.

\section{RESULTS AND DISCUSSION}

Figure 2 shows the return loss characteristics of chip antenna. The calculated resonant frequency of chip antenna is $5.27 \mathrm{GHz}$. The calculated resonant frequency agrees well with the measured result. The length of patch conductor $(6 \mathrm{~mm})$ is smaller than a quarter wavelength. Therefore, the resonant frequency of chip antenna is compared with that of probe-fed shorted microstrip antenna on the same chip. Figure 3 shows the structure of probe-fed shorted microstrip antenna to be mounted on the lower dielectric substrate. The second resonant frequency $5.925 \mathrm{GHz}$ of chip antenna is close to the resonant frequency of probe-fed shorted microstrip antenna.

Figure 4(a) and (b) show the electric field distributions of chip antenna in the vertical plane 
including the central monopole at the first and second resonant frequencies $5.27 \mathrm{GHz}$ and $5.925 \mathrm{GHz}$, respectively. Figure 5 shows the electric field distribution of probe-fed microstrip antenna in the vertical plane including the feeding probe. At the first resonant frequency $5.27 \mathrm{GHz}$ of chip antenna, the electric field is strongly excited at the region between the feeding monopole and the edge of patch conductor. Due to this fringing field, the effective size of this antenna becomes larger. Therefore, the resonant frequency of chip antenna becomes lower than that of probe-fed microstrip antenna.

Figure 6 and 7 show the calculated and measured electric field radiation patterns of chip antenna at the resonant frequency, respectively. The calculated radiation patterns agree well with the measured results.

\section{CONCLUSION}

The shorted microstrip antenna on the parallelepiped rectangular chip has been numerically and experimentally analyzed. This antenna is electromagnetically excited by three monopoles connected on the microstrip line on the lower dielectric substrate. Due to the fringing field between the feeding monopoles and the patch conductor, the resonant frequency of chip antenna becomes lower than the probe-fed shorted microstrip antenna.

\section{REFERENCES}

[1] M. Taguchi, R. Nakamura, H. Shimoda and K. Tanaka, "Rectangular microstrip antenna excited by monopole antenna on finite dielectric substrate," Proc. of 2002 Interim International Symposium on Antennas and Propagation, 2A2-2, Nov. 2002.

[2] M. Taguchi, T. Okajima, H. Shimoda and K. Tanaka, "Shorted rectangular microstirp antenna on dielectric chip for 5.2 GHz Wireless LAN," Proc. Of 2004 IEEE Antennas and Propagation Society International Symposium, vol.4, pp.3812-3815, June 2004.

[3] "Fidelity User's manual Release 3," Zeland Software, Inc., April 2000.

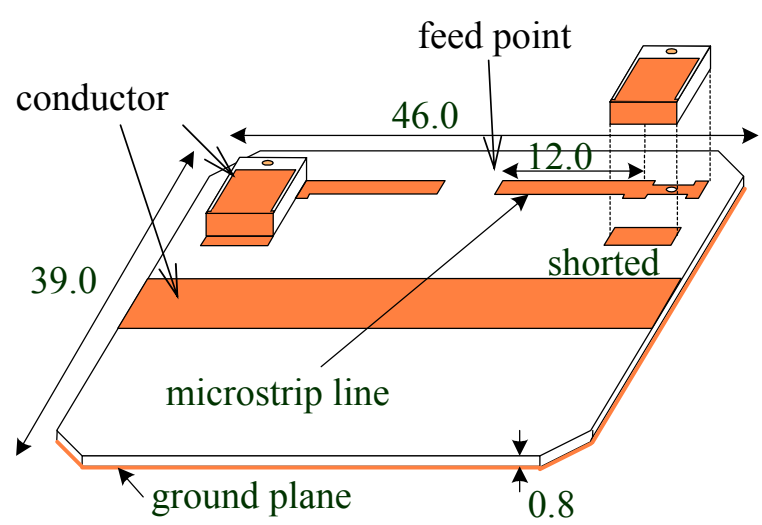

(a) Structure of chip antenna on lower dielectric substrate

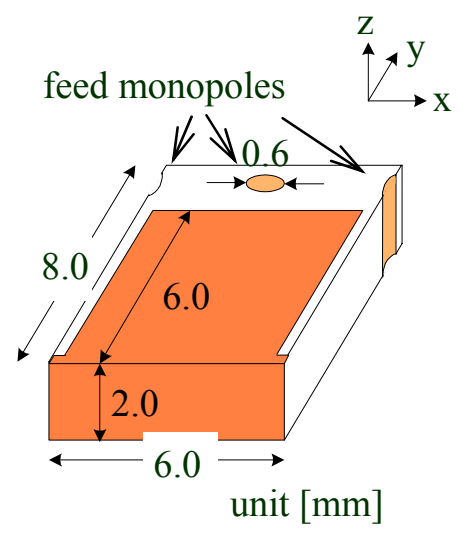

(b) Enlargement of chip

Figure 1 Chip antenna 

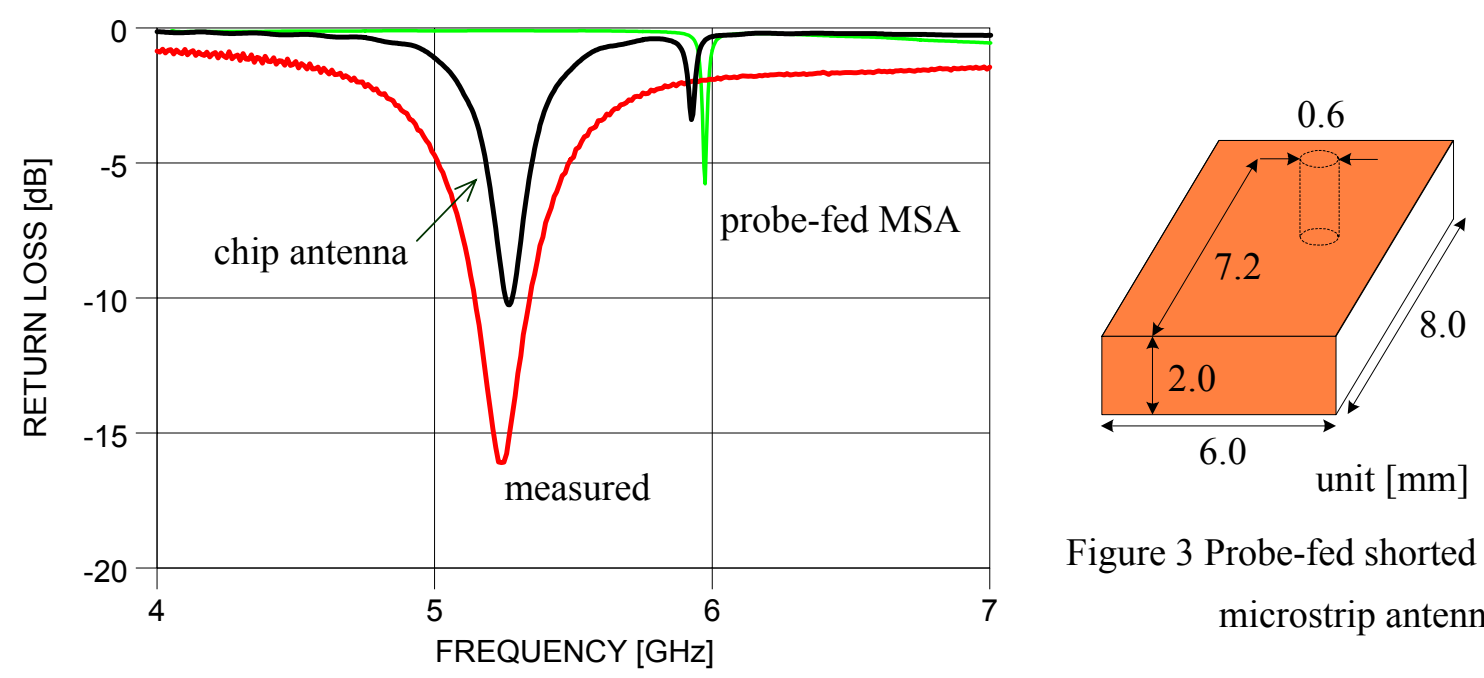

Figure 3 Probe-fed shorted microstrip antenna

Figure 2 Return loss characteristics

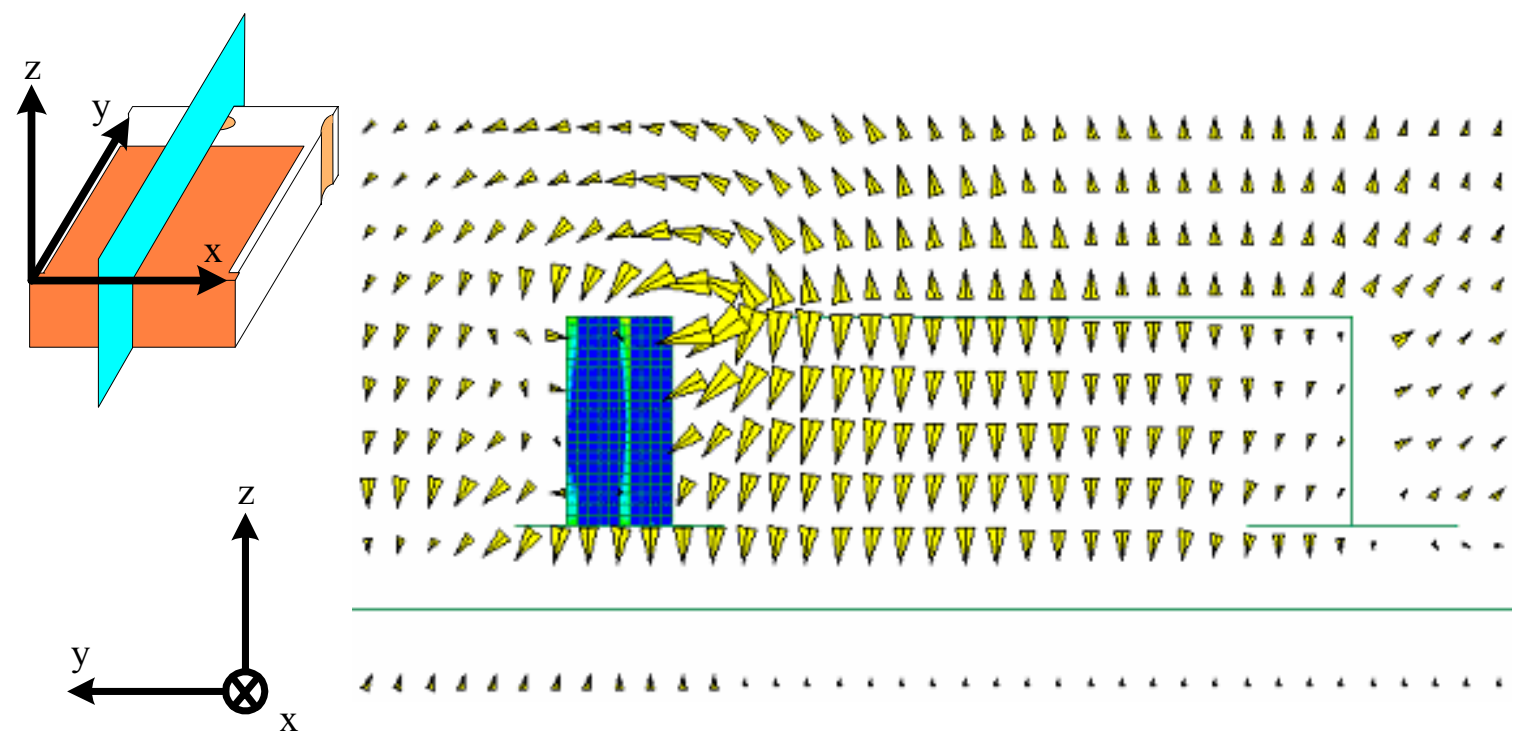

(a) $5.27 \mathrm{GHz}$

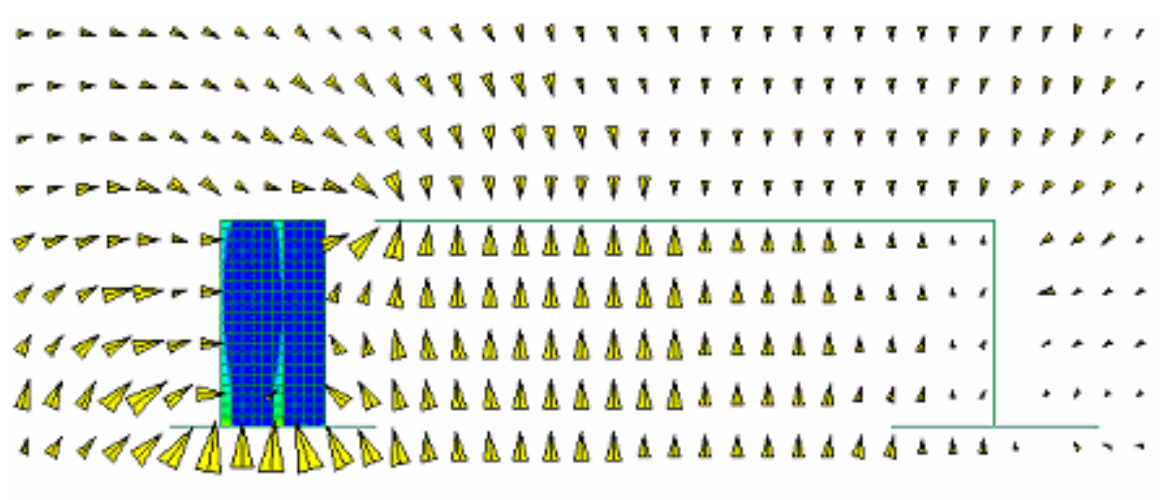

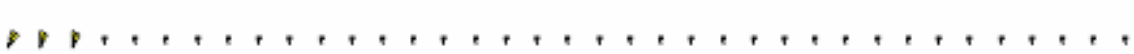

(b) $5.925 \mathrm{GHz}$

Figure 4 Electric field distribution of chip antenna in vertical plane including central monopole. 


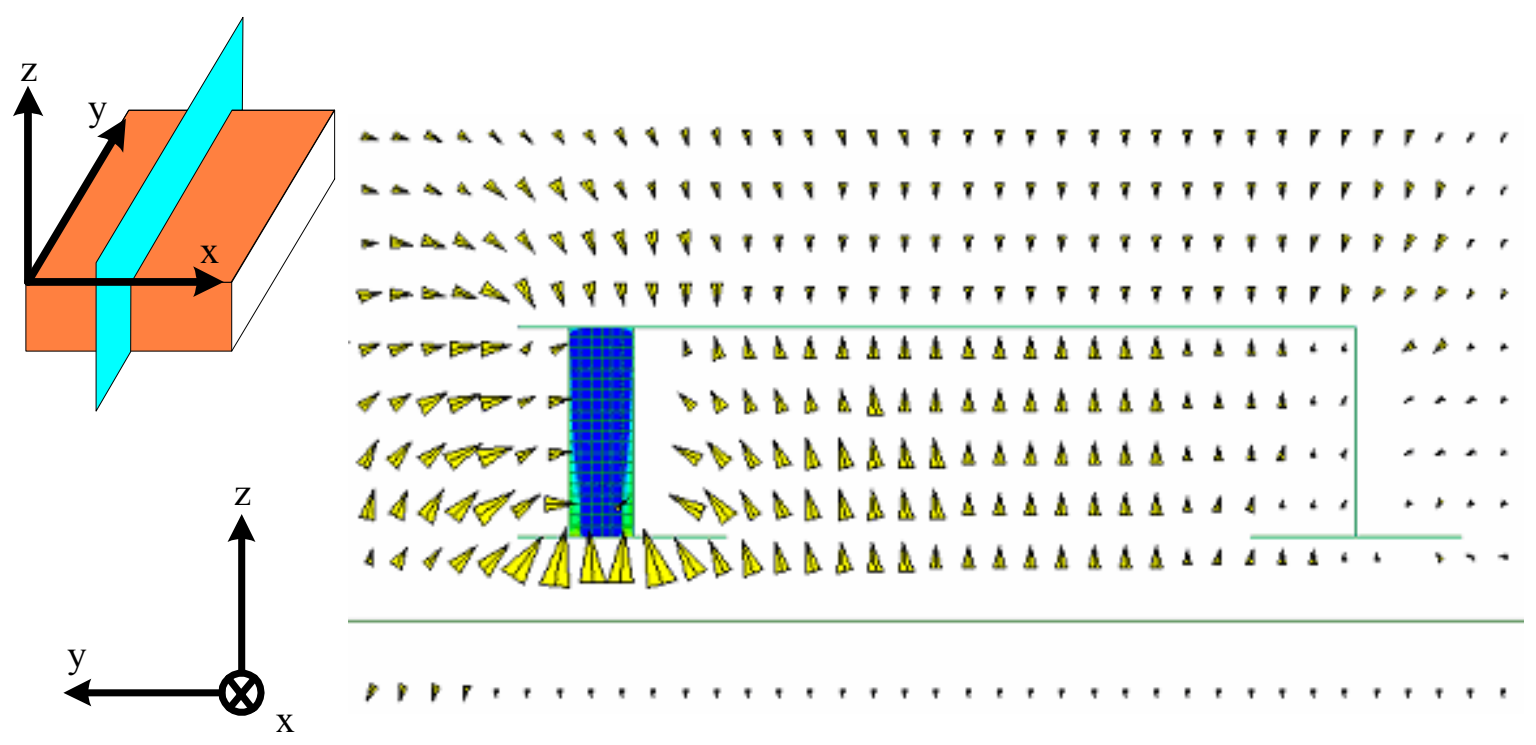

Figure 5 Electric field distribution of probe-fed microstrip antenna in vertical plane including probe at $5.975 \mathrm{GHz}$.

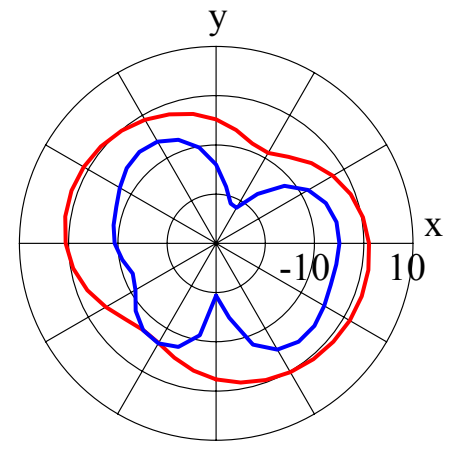

$\theta$-component

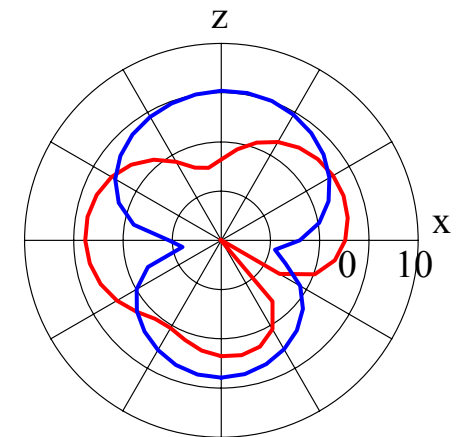

$\phi$-component

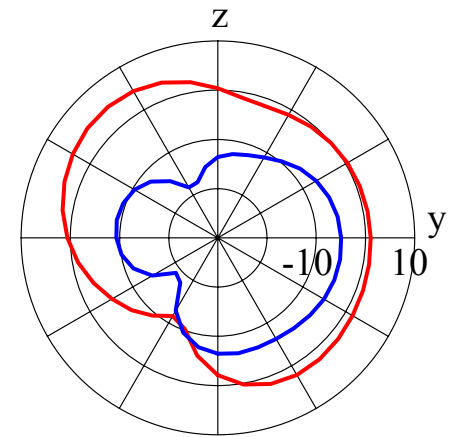

unit $[\mathrm{dB}]$

Figure 6 Calculated electric field radiation patterns.

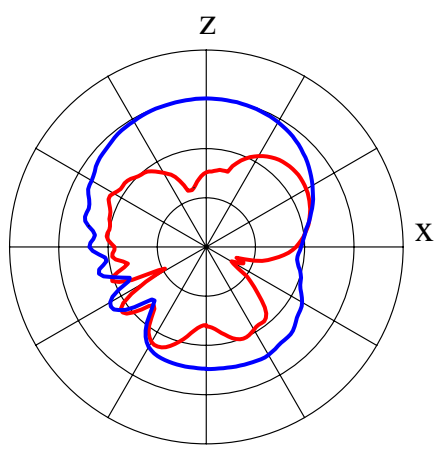

$\theta$-component

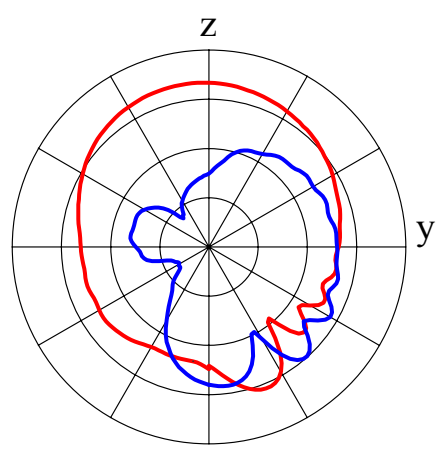

$\phi$-component

Figure 7 Measured electric field radiation patterns. 